\title{
Surgical Outcome of Extra-hepatic Cholangiocarcinoma
}

\author{
Michiaki Unno, Kei Nakagawa, Hiroki Hayashi, Koji Fukase, Fuyuhiko Motoi, Takashi Kamei, \\ Takeshi Naitoh
}

\author{
Corresponding author: \\ Michiaki Unno, M.D., Ph.D \\ Professor and Chairman \\ Department of Surgery, Tohoku University \\ Graduate School of Medicine \\ 1-1, Seiryo-machi, Aoba-ku, Sendai, \\ 980-8574, Japan \\ Tel: +81-22-717-7201 \\ Fax: +81-22-717-7209 \\ E-mail: m_unno@surg.med.tohoku.ac.jp
}

Received: 05.06,2018

Accepted: 24.06.2018
Department of Surgery, Tohoku University Graduate School of Medicine, Sendai, Japan

\begin{abstract}
Extrahepatic cholangiocarcinoma is divided into two compartments, namely perihilar cholangiocarcinoma and distal cholangiocarcinoma. Hemihepatectomy and caudate lobectomy with resection of extrahepatic bile duct is usually performed for patients with perihilar cholangiocarcinoma, whereas pancreaticoduodenectomy is performed for distal cholangiocarcinoma. As pancreaticoduodenectomy has been a well-established and more commonly performed operation, distal cholangiocarcinoma was thought to have better outcomes as compared to perihilar cholangiocarcinoma. However, both cholangiocarcinomas originate from the bile duct epithelium and it is not clear if the malignant potential of perihilar cholangiocarcinoma and distal cholangiocarcinoma are different or not. Therefore, we analyzed 162 perihilar and 98 distal cholangiocarcinoma in our institution from 2002 to 2011 , to clarify the actual overall survival, disease-free survival, and prognostic factors. The actual 5-year overall survivals of perihilar and distal cholangiocarcinoma were $39.8 \%$ and $45.2 \%$, respectively, and actual 5-year disease-free survivals were $29.6 \%$ and $38.4 \%$, respectively. There are no significant differences in overall survival and disease-free survival between perihilar and distal cholangiocarcinoma. In both cholangiocarcinomas groups independent prognostic factors were distant metastasis, lymph node metastases, perineural invasion, and lack of $\mathrm{R} 0$ resection. In conclusion, the oncological outcomes of perihilar and distal cholangiocarcinoma are similar poor long-term outcome for both groups. Effective neoadjuvant and adjuvant therapies must be established to improve patients' prognosis.
\end{abstract}

Key word: distal cholangiocarcinoma, perihilar cholangiocarcinoma, biliary tract cancer, prognostic factor, overall survival

\section{BACKGROUND}

Extrahepatic cholangiocarcinoma is associated with poor prognosis and an over-all survival rate of 20-50 \% (1,2). Extrahepatic cholangiocarcinoma consists of perihilar cholangiocarcinoma and distal cholangiocarcinoma. The surgical treatment for these two types of cholangiocarcinoma is quite different. In general, patients with perihilar cholangiocarcinoma undergo hemihepatecotomy with caudate lobectomy and resection of the extrahepatic bile duct $(3,4)$. Patients with distal cholangiocarcinoma usually undergo pancreaticodudenectomy (5). Because the operation for perihilar cholangiocarci- 
noma is more technically demanding, it is thought that the surgical outcome of perihilar cholangiocarcinoma might be inferior compared to that of distal cholangiocarcinoma. Moreover, both cholangiocarcinomas originate from the epithelium of the bile duct and it is not clear if the malignant potential of perihilar cholangiocarcinoma is different from that of distal cholangiocarcinoma. In this paper, we describe our experience for resection of perihilar and distal cholangiocarcinoma. We compare the two types of extrahepatic cholangiocarinoma to clarify similarities and differences of clinicopathologic characteristics and surgical outcome at a single institution.

\section{PATIENTS AND METHODS}

In this retrospective study, we reviewed all medical records for extrahepatic cholangiocarcinoma. From January 2002 to December 2011, we operated 276 consecutive cases of extrahepatic cholangiocarcinoma (excluding ampulla of Vater carcinoma) in our institution, Tohoku University Hospital. To clarify patients' oncological outcome after surgical resection, we analyzed 260 cases of extra-hepatic cholangiocarcinoma after exclusion of 16 cases in-hospital death. Those patients were divided into two categories, perihilar cholangiocarcinoma and distal cholangiocarcinoma, according to the Japanese classification of Biliary Tract Carcinoma $\left(3^{\text {rd }}\right.$ edition) (6). The primary tumors were classified according to the Japanese classification of biliary tract carcinoma ( $6^{\text {th }}$ edition). We analyzed overall survival and disease-free survival of the perihilar cholangiocarcinoma and distal cholangiocarcinoma with Kaplan-Meier analysis and Cox proportional hazards model.

\section{RESULTS}

According to the Japanese classification of biliary tract cancer ( $3^{\text {rd }}$ edition), 162 (62.3\%) of 260 extrahepatic cholangiocarcinoma were perihilar cholangiocarcinoma and 98 (37.7\%) were distal cholangiocarcinoma.

As shown in table 1,81 of the 260 patients (31.2\%) underwent pancreaticoduodenectomy (PD). Eight patients (3.1\%) underwent right trisectionectomy with caudate lobectomy $(\mathrm{CHx})$ and extrahepatic bile duct resection(BDR). Sixty-eight (26.2\%) received right hepatectomy + $\mathrm{CHX}+\mathrm{BDR}$ and 12 (4.6\%) received right hepatectomy and PD. Six (2.3\%) underwent left trisectionectomy $+\mathrm{CHx}+\mathrm{BDR}, 56$ (21.5\%) underwent left hepatectomy $+\mathrm{CHx}+\mathrm{BDR}$, and 9 (3.5\%) underwent left hepatectomy + PD. Remaining 20 patients (8.5\%)
Table 1

\begin{tabular}{lcc}
\hline Operation & \multicolumn{2}{c}{$\mathbf{n}$} \\
\hline Pancreaticoduodenectomy (PD) & 81 & $31.2 \%$ \\
\hline Right trisectionectomy & 8 & $3.1 \%$ \\
\hline Right hepatectomy & 68 & $26.2 \%$ \\
\hline Right hepatectomy with PD & 12 & $4.6 \%$ \\
\hline Left trisectionectomy & 6 & $2.3 \%$ \\
Left hepatectomy & 56 & $21.5 \%$ \\
Left hepatectomy with PD & 9 & $3.5 \mathrm{z}$ \\
\hline Others & 20 & $8.5 \%$ \\
\hline Total & 260 & $100 \%$ \\
\hline
\end{tabular}

underwent other operation, such as resection of bile duct.

\section{Patients characteristics}

Of the 162 patients with perihilar cholangiocarcinoma, 111 (68.5\%) were male and 51 (31.5\%) were female (table 2). The mean age was 67.0 years (range 41-82). On the other hand, of 98 patients with distal cholangiocarcinoma, $66(67.3 \%)$ were male and $32(32.7 \%)$ were female. The mean age was 69.5 years (range 15-83). There are no significant differences of sex and age between perihilar and distal cholangiocarcinoma.

However, the histopathological status was quite different $(p<0.0001)$. In perihilar cholangiocarcinoma, most patients presented with a pT2 $(n=80,49.3 \%)$ or pT4 tumor $(n=52,35.2 \%)$, whereas in distal cholangiocarcinoma, pT3 tumors were most frequently found ( $n=59,60.2 \%$ ) with no patient presenting with a pT4 tumor.

Histopathologically positive lymph nodes were found in $46.9 \%$ in perihilar cholangiocarcinoma and $31.6 \%$ in distal cholangiocarcinoma $(p=0.0145)$. The patients who underwent preoperative biliary drainage were $77.2 \%$ in perihilar cholangiocarcinoma and 88.8 $\%$ in distal cholangiocarcinoma $(p=0.0028)$. R0 resection rate was $71.6 \%$ in perihilar cholangiocarcinoma and $85.7 \%$ in distal cholangiocarcinoma $(p=0.0175)$.

\section{Overall survival}

The median follow-up time was $\mathbf{3 8 . 2}$ months for all cohort and the median follow-up time of survival cases was 68.7 months. The actual 5-year overall survival rates were $39.8 \%$ in perihilar cholangiocarcinoma and $45.2 \%$ in distal cholangiocarcinoma (fig. 1). The median survival time were 42.1 months in perihilar cholangio- 
Table 2

\begin{tabular}{|c|c|c|c|}
\hline & Perihilar $(n=162)$ & Distal $(n=98)$ & $p$ value \\
\hline Man / Woman & $111(68.5 \%) / 51(31.5 \%)$ & $66(67.3 \%) / 32(32.7 \%)$ & n.s. \\
\hline $\begin{array}{l}\text { Age } \\
\text { <Median> }\end{array}$ & $\begin{array}{r}67.0 \\
(41-82) \\
\end{array}$ & $\begin{array}{r}69.5 \\
(15-83)\end{array}$ & n.s. \\
\hline pT $(1 / 2 / 3 / 4)$ & $8 / 80 / 17 / 57$ & $10 / 29 / 59 / 0$ & $<0.0001$ \\
\hline pN $(0 / 1)$ & $86(53.1 \%) / 76(46.9 \%)$ & $67(68.4 \%) / 31(31.6 \%)$ & 0.0145 \\
\hline Billiary drainage & $125(77.2 \%)$ & $87(88.8 \%)$ & 0.0028 \\
\hline $\mathrm{R}(0 / 1 / 2)$ & $116(71.6 \%) / 42$ / 4 & $84(85.7 \%) / 12$ / 2 & 0.0175 \\
\hline
\end{tabular}

carcinoma and 51.9 months in distal cholangiocarcinoma. There is no significant difference between perihilar and distal cholangiocarcinoma.

\section{Disease-free survival}

As shown in fig. 2, the actual 5-year disease-free survival (DFS) rates were $29.6 \%$ in perihilar cholangiocarcinoma and $38.4 \%$ in distal cholangiocarcinoma. There is no significant difference between two cholangiocarcinoma.

\section{Multivariate analysis}

The results of multivariate analysis by Cox proportional hazards model are shown in fig. 3. On multivariate analysis presence of distant metastases (HR 3.26; $p<0.0001$ ), perineural invasion (HR 2.65; $p=0.0003$ ), lymph node metastasis (HR 1.63; $\mathrm{p}=0.0067)$, and positive resection margin (HR 1.64; $p=0.0108$ ) were associated withpoor survival. There were no significant association of pathological grading, T stage, preoperative biliary drainage, adjuvant chemotherapy, severe complication (ClavianDindo $>3$ ) or tumor location with survival.

Survival was significantly difference between patients with pNO and pN1 ( $<<0.0001$; log-rank test). The 5-year survival rate of the patients with lymph node metastases was $20.2 \%$ (median survival 27.3 months) was $55.8 \%$ (median survival 75.0 months) in patients without lymph node metastases (fig. 4). The 5-year

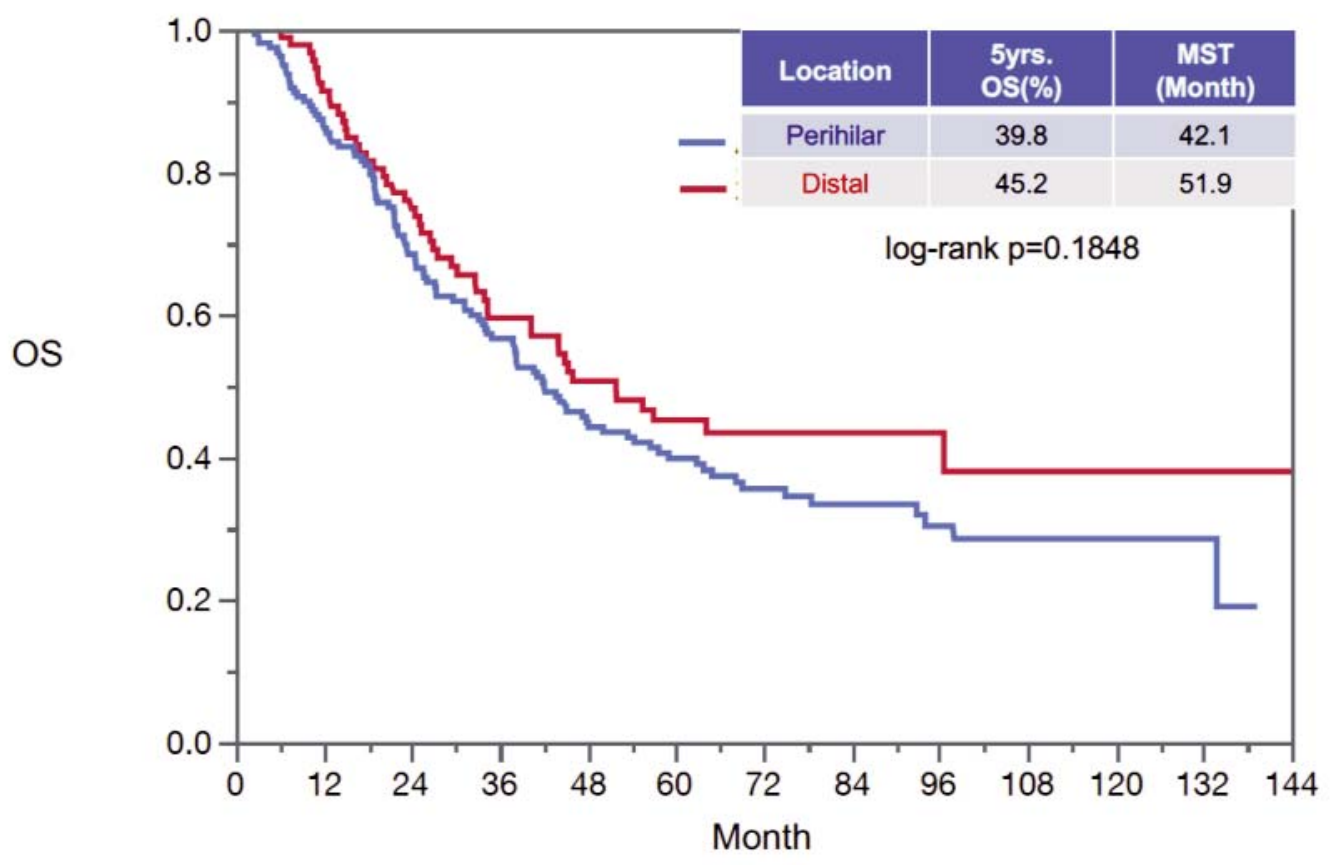

Figure 1 - Overall Kaplan-Meier curves of perihilar cholangiocarcinoma and distal cholangiocarcinoma. The outcome of distal cholangiocarcinoma was superior compared to perihilar cholangiocarcinoma, but there is no significant difference $(p=0.1848$ by log-rank) 


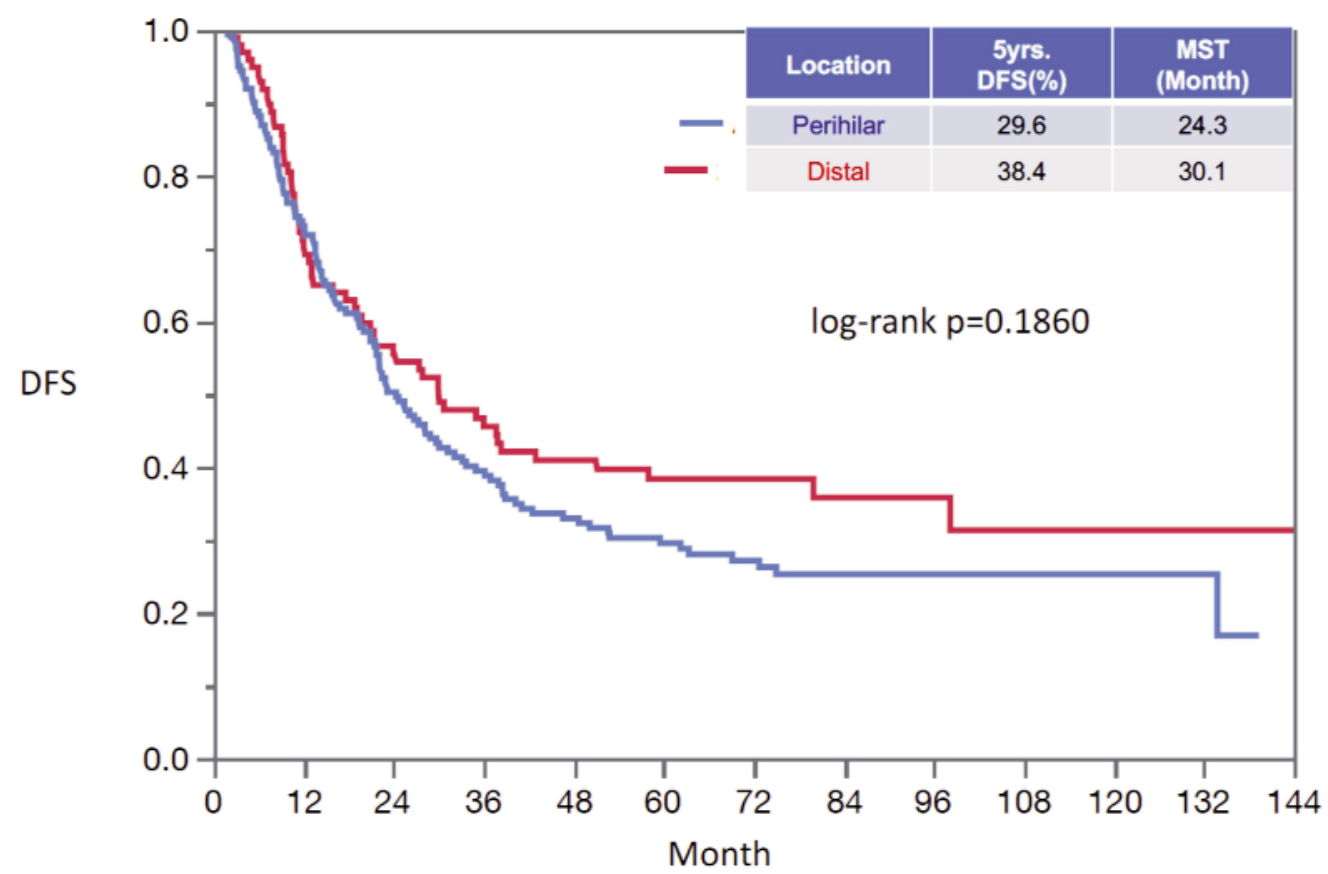

Figure 2 - Disease free survival curves of perihilar cholangiocarcinoma and distal cholangiocarcinoma

survival of the patients with R0 resection was $46.5 \%$ (median survival 54.4 months), whereas the 5 -year survival after R1 and R2 resection were $26.6 \%$ (median survival 32.1 months)and 0\% (median survival 19.1 months), respectively (fig. 5).

\section{DISCUSSION}

In this retrospective study, we analyzed the oncological outcome of extrahepatic cholangiocarcinoma. Surgical resection is the only curative therapy for extrahepatic cholangiocarcinoma. The standard chemotherapy for unresectable extrahepatic cholangiocarcinoma is gemcitabine plus cisplatin with a median survival of 11.7 months and a 5-year survival rate of $0 \%$ (7).

Prior to this retrospective study, long-term outcome of distal cholangiocarcinoma was believed to be

10

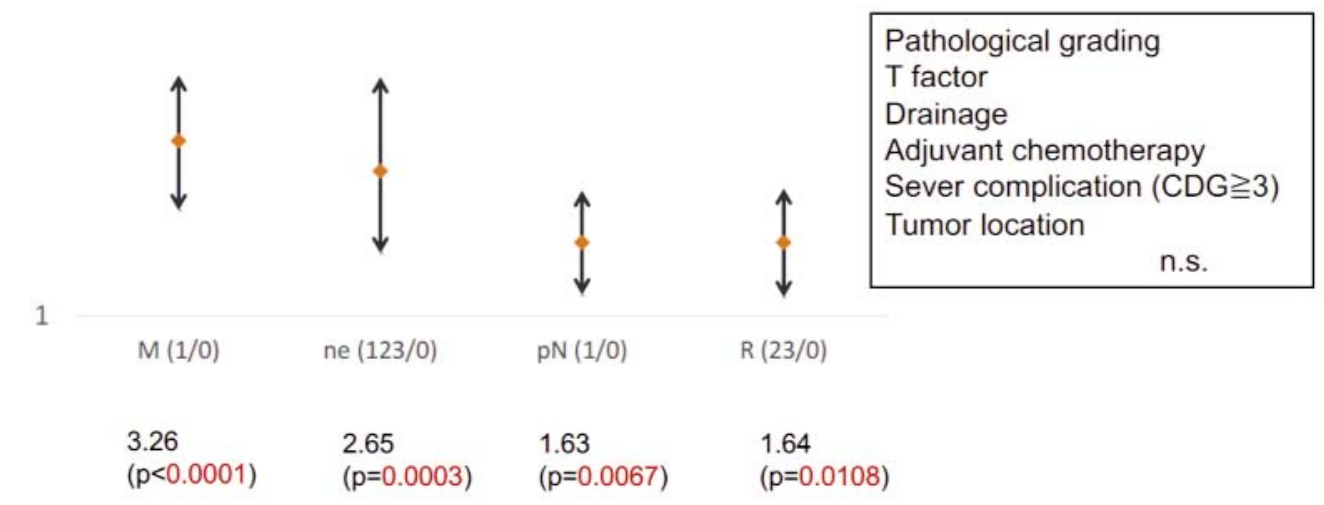

0.1

Figure 3 - The results of Cox harzards model for prognostic factors for extrahepatic cholangiocarcinoma 


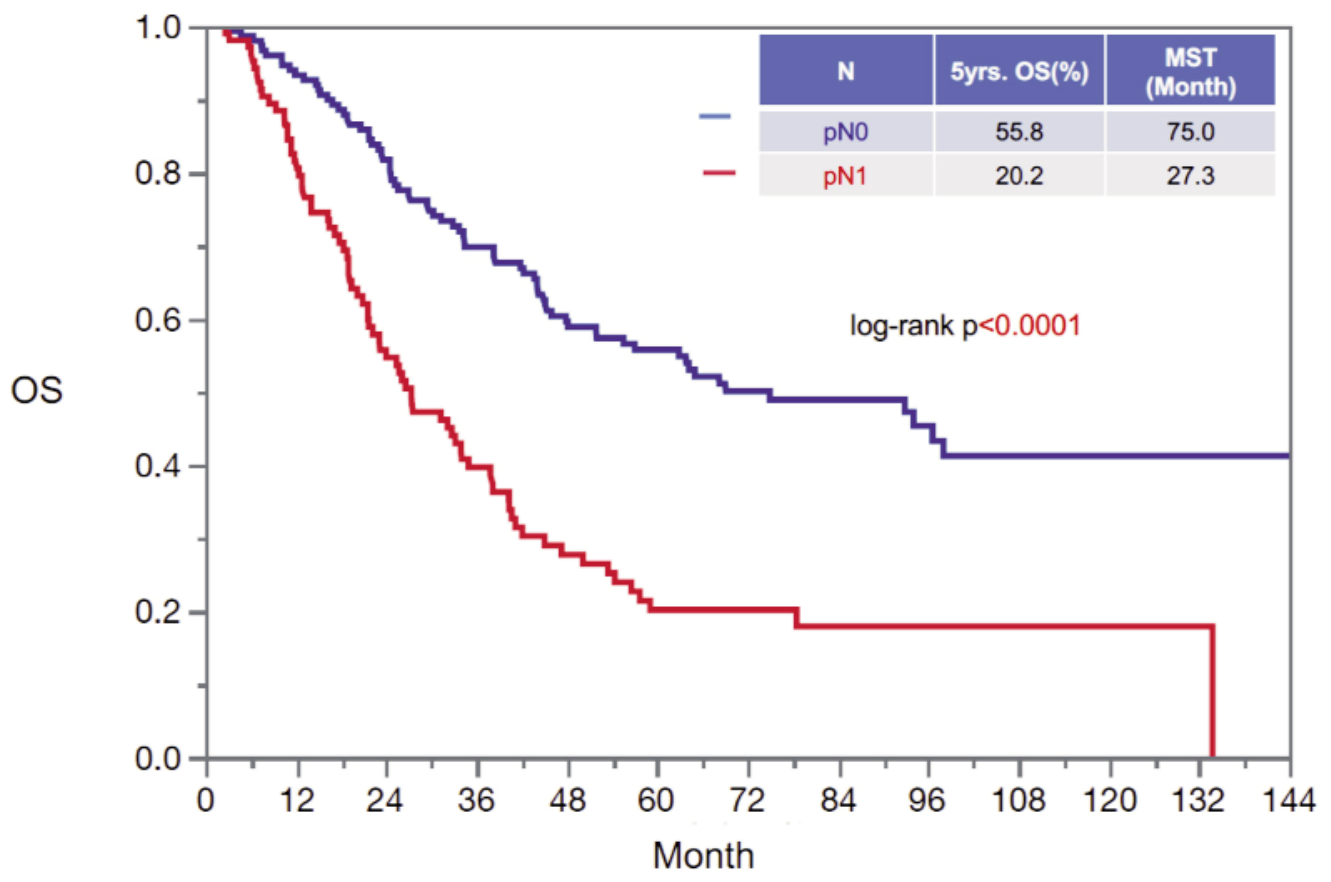

Figure 4 - Overall survivals in patients with pNO and pN1

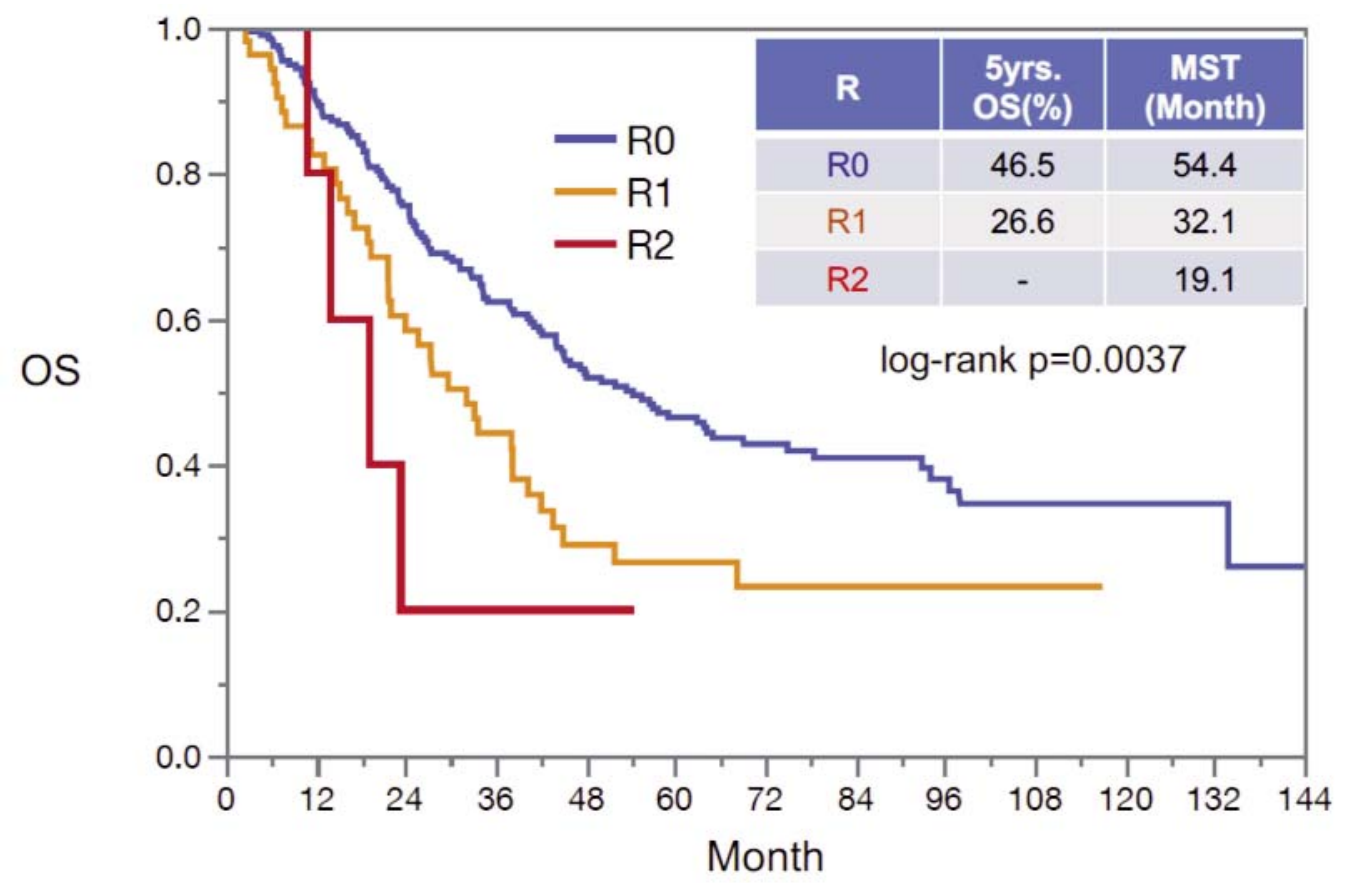

Figure 5 - Overall survivals curves according to the curability (R0/R1/R2)

superior to that of perihilar cholangiocarcinoma, because of a more complex surgical approach is required to achieve complete tumor resection in patients with perihilar tumors. Indeed, the percentage of patients with negative lymph nodes and $\mathrm{RO}$ resection was higher in distal cholangiocarcinoma than in perihilar cholangiocarcinoma. However, neither overall survival nor disease-free survival were significantly different between perihilar cholangiocarcinoma and distal cholangiocarcinoma. 
It needs to be noted that the staging system of perihilar cholangiocarcinoma and distal carcinoma is different. For example, pT4 stage in perihilar cholangiocarcinoma includes pT4a, which is Bismuth-Corlette classification type 4, and pT4b, which presents invasion to both or contralateral portal vein or hepatic artery. Many surgeons pointed out that most cases of pT4a and $\mathrm{pT} 4 \mathrm{~b}$ perihilar cholangiocarcinoma were resectable (8). On the other hand, pT4 distal cholangiocarcinoma invaded the celiac, common hepatic artery or superior mesenteric artery. pT4 distal cholangiocarcinoma is commonly considered an unresectable tumor.

In this study, the multivariate analysis revealed that the prognostic factors for extrahepatic cholangiocarcinoma were distant metastasis, perineural invasion, lymph node metastasis, and resection margin. Lymph node metastasis was one of the most important prognostic factors of extrahepatic cholangiocarcinoma $(4,9)$. Moreover, the number of involved nodes as a prognostic factor in distal cholangiocarcinoma (10) and perihilar cholangiocarcinoma (11). In our study, overall survival of patients with positive lymph nodes was significantly lower than those with negative lymph nodes. Lymph node metastasis was a strong prognostic factor in both perihilar and distal cholangiocarcinoma.

Some authors have reported perineural invasion as significant prognostic factor in cholangiocarcinoma (1214). In line with these data in the present study, survival of patients with perineural invasion was worse than that of patients without perineural invasion. This association remained to be significant on multivariate analysis.

Many studies have reported that RO resection is necessary for long-term survival $(4,15,16)$. In our previous study, we revealed that RO resection was among the strongest predictive factors and we concluded that the surgeon's goal for a patient's longterm survival is complete resection of the tumor with negative margins (3).

\section{CONCLUSION}

In conclusion, surgical outcome of both perihilar cholangiocarcinoma and distal cholangiocarcinoma are similar in this single institutional analysis. Perineural invasion, lymph node metastasis and positive resection margin are prognostic factors in patients who underwent resection with curative intent.

\section{Conflicts of interest}

The authors declare that there are no conflicts of interest.

\section{REFERENCES}

1. Rizvi S, Gores GJ. Pathogenesis, diagnosis, and management of cholangiocarcinoma. Gastroenterology. 2013;145(6):1215-1229.

2. DeOliveira ML, Cunningham SC, Cameron JL, Kamangar F, Winter JM, Lillemoe KD, et al. Cholangiocarcinoma: thirty-one-year experience with 564 patients at a single institution. Ann Surg. 2007;245(5) :755-62.

3. Unno M, Katayose Y, Rikiyama T, Yoshida H, Yamamoto K, Morikawa $\mathrm{T}$, et al. Major hepatectomy for perihilar cholangiocarcinoma. J Hepatobiliary Pancreat Sci. 2010;17(4):463-9.

4. Nagino M, Ebata T, Yokoyama Y, Igami T, Sugawara G, Takahashi $\mathrm{Y}$, et al. Evolution of surgical treatment for perihilar cholangiocarcinoma: a single-center 34-year review of 574 consecutive resections. Ann Surg. 2013;258(1):129-40.

5. Suzuki M, Unno M, Oikawa M, Endo K, Katayose Y, MATSUNO S. Surgical treatment and postoperative outcomes for middle and lower bile duct carcinoma in Japan--experience of a single institute. Hepatogastroenterology. 2000;47(33):650-657.

6. Miyazaki M, Ohtsuka M, Miyakawa S, Nagino M, Yamamoto M, Kokudo $\mathrm{N}$, et al. Classification of biliary tract cancers established by the Japanese Society of Hepato-Biliary-Pancreatic Surgery: 3(rd) English edition. J Hepatobiliary Pancreat Sci. 2015;22(3):181-96.

7. Valle $\mathrm{J}$, Wasan $\mathrm{H}$, Palmer $\mathrm{DH}$, Cunningham $\mathrm{D}$, Anthoney $\mathrm{A}$, Maraveyas A, et al. Cisplatin plus gemcitabine versus gemcitabine for biliary tract cancer. N Engl J Med. 2010;362(14):1273-81.

8. Ebata T, Kosuge T, Hirano S, Unno M, Yamamoto M, Miyazaki M, et al. Proposal to modify the International Union Against Cancer staging system for perihilar cholangiocarcinomas. $\mathrm{Br} \mathrm{J}$ Surg. 2014;101(2):79-88.

9. Cheng Q, Luo X, Zhang B, Jiang X, Yi B, Wu M. Distal bile duct carcinoma: prognostic factors after curative surgery. A series of 112 cases. Ann Surg Oncol. 2007;14(3):1212-1219.

10. Kiriyama M, Ebata T, Aoba T, Kaneoka Y, Arai T, Shimizu Y, et al. Prognostic impact of lymph node metastasis in distal cholangiocarcinoma. Br J Surg. 2015;102(4):399-406.

11. Aoba T, Ebata T, Yokoyama Y, Igami T, Sugawara G, Takahashi Y, et al. Assessment of nodal status for perihilar cholangiocarcinoma: location, number, or ratio of involved nodes. Ann Surg. 2013; 257(4):718-25

12. Kim HJ, Kim CY, Hur YH, et al. The prognostic factors for survival after curative resection of distal cholangiocarcinoma: perineural invasion and lymphovascular invasion. Surg Today. 2014;44(10): 1879-1886.

13. Murakami Y, Uemura K, Sudo T, Hashimoto Y, Kondo N, Nakagawa $\mathrm{N}$, et al. Perineural invasion in extrahepatic cholangiocarcinoma: prognostic impact and treatment strategies. J Gastrointest Surg. 2013;17(8):1429-39.

14. Neuhaus P, Jonas S, Bechstein WO, Lohmann R, Radke C, Kling N, et al. Extended resections for hilar cholangiocarcinoma. Ann Surg. 1999;230(6):808-18-discussion 819.

15. Jarnagin WR, Fong Y, DeMatteo RP, Gonen M, Burke EC, Bodniewicz BS J, et al. Staging, resectability, and outcome in 225 patients with hilar cholangiocarcinoma. Ann Surg. 2001;234(4):507-17; discussion 517-9.

16. Sakamoto Y, Kosuge T, Shimada K, Sano T, Ojima H, Yamamoto J, et al. Prognostic factors of surgical resection in middle and distal bile duct cancer: An analysis of 55 patients concerning the significance of ductal and radial margins. Surgery. 2005;137(4):396-402. 